\title{
Inter-hemispheric EEG coherence analysis in Parkinson's disease: Assessing brain activity during emotion processing
}

R. Yuvaraj *, M. Murugappan, Norlinah Mohamed Ibrahim, Kenneth Sundaraj, Mohd Iqbal Omar, Khairiyah Mohamad, R. Palaniappan, M. Satiyan

\begin{abstract}
Affiliations
R. Yuvaraj, School of Mechatronic Engineering, University Malaysia Perlis ; M. Murugappan, School of Mechatronic Engineering, University Malaysia Perlis ; Norlinah Mohamed Ibrahim, Head, Neurology Unit, Department of Medicine, UKM Medical Center; Kenneth Sundaraj, School of Mechatronic Engineering, University Malaysia Perlis ; Mohd Iqbal Omar, School of Mechatronic Engineering, University Malaysia Perlis; R. Palaniappan, Faculty of Science and Engineering, University of Wolverhampton, Telford, UK Khairiyah Mohamad, , Neurology Unit, Department of Medicine, UKM Medical Center ; M. Satiyan, Research scholar, School of Mechatronic Engineering, University Malaysia Perlis ;
\end{abstract}

*Correspondence concerning this article should be addressed to: R. Yuvaraj, School of Mechatronic Engineering, Campus UluPauh, Arau, Perlis, 02600, Malaysia E-mail: yuva2257@gmail.com; Tel: +6-014-6011990; Fax: 04-988-5167

Keywords: emotion; inter-hemispheric coherence; electroencephalography; Parkinson's disease. 


\begin{abstract}
Objective: Parkinson's disease (PD) is not only characterized by its prominent motor symptoms but it also associated with disturbances in cognitive and emotional functioning. The objective of the present study was to investigate the influence of emotion processing on inter-hemispheric electroencephalography (EEG) coherence in PD.
\end{abstract}

Methods: Multimodal emotional stimuli (happiness, sadness, fear, anger, surprise, and disgust) were presented to $20 \mathrm{PD}$ patients and 30 age-, education level-, and gender-matched healthy controls (HC) while EEG was recorded. Inter-hemispheric coherence was computed from seven homologous EEG electrode pairs (AF3-AF4, F7-F8, F3-F4, FC5-FC6, T7-T8, P7-P8, and O1O2) for delta, theta, alpha, beta, and gamma frequency bands. In addition, subjective ratings were obtained for a representative of emotional stimuli.

Results: Interhemispherically, PD patients showed significantly lower coherence in theta, alpha, beta, and gamma frequency bands than $\mathrm{HC}$ during emotion processing. No significant changes were found in the delta frequency band coherence. We also found that PD patients were more impaired in recognizing negative emotions (sadness, fear, anger, and disgust) than relatively positive emotions (happiness and surprise). Behaviorally, PD patients did not show impairment in emotion recognition as measured by subjective ratings.

Conclusion: These findings suggest that PD patients may have an impairment of interhemispheric functional connectivity (i.e., a decline in cortical connectivity) during emotion processing.

Significance: This study may increase the awareness of EEG emotional response studies in clinical practice to uncover potential neurophysiologic abnormalities.

Keywords: emotion; inter-hemispheric coherence; electroencephalography; Parkinson's disease. 


\section{INTRODUCTION}

The neuropathological hallmark of Parkinson's disease (PD) is the progressive loss of dopamine neurons in the substantia nigra pars compacta and the subsequent striatal dopamine deficiency, which leads to a cascade of dysfunctions in the basal ganglia and dopaminergic pathways (Obeso et al. 2008; Péron et al. 2012). In addition to well-known motor symptoms, such as resting tremor, rigidity, bradykinesia, and postural instability (Chen et al. 2013; Savitt et al. 2006), PD is also associated with disturbances in cognitive functions (Levin et al. 1989; Pillon et al. 1996; Mohr et al. 1990), neuropsychiatric status (Celesia and Wanamaker 1972), language (Bastiaanse and Leenders 2009) and particularly, in emotion recognition (Gray and TickleDegnen 2010; Péron et al. 2012).

Individuals with PD show impairments in the ability to recognize emotions from facial expression (Clark et al. 2008; Dujardin et al. 2004; Lawrence et al. 2007; Sprengelmeyer et al. 2003), speech prosody, (Dara et al. 2008; Pell and Leonard 2003; Yip et al. 2003; Paulmann and Pell 2010) and show reduced startle reactivity to highly arousing unpleasant pictures (Bowers et al. 2006; Miller et al. 2009). There is limited event related potential (ERP) evidence that early processing of emotional prosody (mismatch negativity; Schröder et al. 2006) and faces (early posterior negativity; Wieser et al. 2012) may be affected in PD. While there are some reports of intact emotion recognition (Madeley et al. 1995; Pell and Leonard 2005; Garrido-Vásquez et al. 2012; Adolphs et al. 1998; Caekebeke et al. 1991; Wieser et al. 2012; Dujardin et al. 2004), others have documented impairments in recognizing some of the basic emotions (anger, fear, disgust, happiness, sadness, and surprise) (Lawrence et al. 2007; Suzuki et al. 2006). Most recently, lateralization (left versus right) of emotion recognition in PD has become controversial. For example, Clark et al. (2008) reported no asymmetry effects on explicit emotion 
categorization (Clark et al. 2008) while Ariatti et al. (2008) and Yip et al., (2003) reported problems in categorizing disgust prosody in patients with predominantly right-sided motor symptoms (Ariatti et al. 2008; Yip et al. 2003). Additionally, Ventura et al. (2012) reported that predominantly left-sided patients exhibit problems in the recognition of sadness emotion (Ventura et al. 2012). Finally, it is not yet clear whether deficits in emotion recognition are confined to one stimulus modality (i.e., facial expressions; Clark et al. 2008) or more (facial displays and prosody; Ariatti et al. 2008; Dara et al. 2008), as more generally diminished emotion recognition abilities in PD patients in different communication channels have been recently reported (facial displays, voices, and verbs; Paulmann and Pell 2010). Taken together, experimental evidence so far supports the view of impairments in emotion processing in PD. In addition, most of the above mentioned studies were based on behavioral responses to emotional stimuli (i.e., participants were asked to match, identify, judge, or rate the emotional stimuli) and few studies have measured ERPs.

The underlying cause of these problems in processing of emotional information in PD may be the neuropathological changes in many brain areas, which are postulated to play key roles in emotion processing (Kober et al. 2008; Sergerie et al. 2008). These areas include limbic structures, such as the amygdala and the ventral striatum (centrally located within the basal ganglia's limbic loop). Moreover, Tessitore et al. (2002) and Beyer et al. (2007) demonstrated in functional magnetic resonance imaging (fMRI) and structural MRI studies that dopamine levels modulate the amygdala's response in PD patients involving processing of fearful emotional stimuli (Tessitore et al. 2002) and that PD patients show volumetric reductions in the amygdala (Beyer et al. 2007). In addition, Cancelliere and Kertz (1990) reported that patients with cortical lesions who had additional damage to the basal ganglia showed the most pronounced deficits in 
emotional judgments (Cancelliere and Kertesz 1990). Taken together, the neuropathological changes in emotion-related brain areas, the influence of dopamine deficiency on the amygdala as well as the degeneration of basal ganglia and the amygdala might provide an explanation for the observed deficits in emotion processing in PD.

Coherence is a frequency-specific quantitative measure for the similarity of two different signals. Applied to electroencephalographic (EEG) measures, coherence can be interpreted as a quantitative measure of the degree of functional connectivity between distinct brain regions (French and Beaumont 1984). Previous studies have applied coherence to the examination of functional changes associated with the performance of a perceptual or cognitive task, and a coherence measure obtained from spectral EEG analysis is thought to be a useful technique in the assessment of cognitive function (Busk and Galbraith 1975; Shaw et al. 1977; Gasser et al. 1987). There have been numerous EEG studies of PD that have mainly shown the slowing of EEG background activity (Neufeld et al. 1988) as well as, changes in alpha/theta rhythms (Soikkeli et al. 1991). Several previous studies have used EEG coherence analysis to investigate functional changes in numerous disease states in different situations, but the path of change is inconsistent across those states. Certain brain regions and frequency ranges show an increase in coherence in multi-infract dementia (Leuchter et al. 1992), AIDS (Newton et al. 1994), and mild head injury (Thatcher et al. 1989), while decreases are observed in Alzheimer's disease (Leuchter et al. 1992) and depression (Roemer et al. 1992). For PD, only one study (Fonseca et al. 2013) evaluated the relationship between EEG coherence and cognition, and the results indicated an increase in beta frontal-occipital inter-hemispheric coherence in PD patients compared to healthy controls (HC). To date, there have been very few studies that have analyzed EEG coherence during emotion processing in HC participants (Hinrichs and Machleidt 1992; 
Schellberg et al. 1990). Hinrichs and Machleidt (1992) used coherence capability to describe inter-hemispheric coupling effects in order to differentiate between various kinds of emotion; therefore, coherence analysis of EEGs recorded during emotion processing could provide a useful means for assessing emotional impairments in PD patients. To the best of our knowledge, however, no studies have specifically investigated inter-hemispheric synchronization of EEG activity during emotion processing in $\mathrm{PD}$ patients compared to $\mathrm{HC}$.

\section{MATERIALS AND METHODS}

\section{Participants}

Twenty three PD patients and 30 age-, education level-, and gender-matched HC participated in the study. Due to excessive artifacts (movements or closing of the eyes), three participants of the PD group had to be excluded from further analysis, resulting in a sample of 20 PD patients (10 men and 10 women) and $30 \mathrm{HC}$ (13 men and 17 women). The PD patients were recruited from the clinic Neurology outpatient service of the Hospital University Kebangsaan Malaysia (HUKM) medical center in Kuala Lumpur, Malaysia. All patients had been diagnosed with idiopathic PD by a neurologist and were optimally medicated during the testing session (ON state) with a d2-agonist $(\mathrm{n}=18)$; carbidopa/L-dopa $(\mathrm{n}=13)$, monoamine oxidase B (MAO-B) inhibitor $(n=7)$, catechol-O-methyltransferase $($ COMT) inhibitor $(n=5)$, amantadine $(n=5)$, or anticholinergics $(n=3)$. The average duration of $\mathrm{PD}$ (post-diagnosis) in the group was 5.75 years [standard deviation $(\mathrm{SD})=3.52$, range $=1-12$ years]. The severity of motor signs in the patient group could be characterized as mild to moderate; all patients fit Hoehn and Yahr stages (H \& Y) (Hoehn and Yahr 1967) I - III (Stage I = unilateral disease with mild symptoms, Stage II = bilateral involvement, Stage III = bilateral symptoms with postural and gait disturbances) with a mean Unified Parkinson's Disease Rating Scale (UPDRS) (Fahn et al. 1987) motor score of 
$17.05(\mathrm{SD}=3.15)$. None of the patients had coexisting neurological (e.g., epilepsy) or psychiatric disturbances (e.g., major depression or anxiety, psychotic symptoms, etc.) that might independently influence their cognitive functioning.

The HC participants were recruited through the hospital's medical unit community and/or from patient's relatives. Exclusion criteria for $\mathrm{HC}$ participants included any current psychiatric or neurological disorder. Exclusion criteria for both the groups were dementia or depression as indicated by a score of 24 or lower on the Mini-Mental State Examination (MMSE) (Folstein et al. 1975; Wieser et al. 2012) or 18 or higher on the Beck Depression Inventory (BDI) (Beck et al. 1961; Schröder et al. 2006). All participants were right-handed as determined by self-report and confirmed by Edinburgh Handedness Inventory (EHI) (Oldfield 1971). This test consisted of 10 questions asking for the preferred hand for a series of activities (e.g., writing, throwing, using scissors, etc.). All participants reported normal or corrected-to-normal vision, and intact hearing was formally established in all participants by administering a pure tone audiometric screening of both ears to ensure acceptable normal hearing threshold (minimum $30 \mathrm{~dB} \mathrm{HL}$ at $0.5,1,2$, and 4 $\mathrm{kHz}$, for the better hearing). All participants/caretakers gave informed consent before completing the study, which was approved by the Faculty of Medicine, Institutional Review Board of the University Kebangsaang Malaysia for ethical adherence. All participants were paid 50 Malaysian Ringgits for their participation.

Patients and controls were comparable in demographic variables, such as age (PD: mean $(\mathrm{SD})=59.05(5.64)$ years; HC: mean $(\mathrm{SD})=58.43(3.01)$ years; $\mathrm{t}(48)=0.502, p=0.61)$, gender distribution (PD: 10 men, HC: 13 men; $\left.x^{2}(1, \mathrm{~N}=50)=0.21, p=0.68\right)$, and education level (PD: mean $(\mathrm{SD})=10.45(4.8)$ years; HC: mean $(\mathrm{SD})=11.02(3.24)$ years; $\mathrm{t}(48)=-0.62, p=0.51)$. As 
shown in Table 1, PD patients and HC participants did not differ in mean MMSE scores, mean BDI scores as well as mean EHI scores.

\section{Stimulus material}

Acquiring emotional data that corresponds to specific emotional state is challenging because of the subjective nature of the emotions and cognitive dependence of physiological signals which requires the emotional states to be elicited internally in the participant. Until now, most studies on emotion recognition in PD have used only facial, prosodic, or music stimuli (Gray and Tickle-Degnen 2010; Péron et al. 2012; Lima et al. 2013). In addition, a number of emotion induction techniques using pictures, sounds, music, or multimodal approach (combination of audio and visual) have been used to elicit target emotions (Baumgartner et al. 2006; Davidson 1992; Gross and Levenson 1995; Murugappan et al. 2009; Jerritta et al. 2013a; Petrantonakis and Hadjileontiadis 2011) in healthy controls. Among all of these stimuli modalities, researchers have found that multimodal stimuli induce emotions in the participants more naturally and more effectively compared to other modalities (Gross and Levenson 1995; Kim et al. 2004; Murugappan et al. 2009; Wang and Guan 2008). Hence, we utilized a multimodal approach to evoke six basic emotions (happiness, sadness, fear, anger, surprise, and disgust) that are universally accepted.

The emotional stimuli we used were taken from different sources such as the International Affective Picture System (IAPS) database (Lang et al. 1993), International Affective Digitized Sounds (IADS) (Bradley and Lang 2007) database and video clips (e.g., funny animals, wonder activities by humans etc.) collected from various resources on the internet (e.g., YouTube, Facebook, and others) (Jerritta et al. 2013b). The elicitation of emotions such as sadness, fear, and disgust was attained by using affective pictures from IAPS and sounds from IADS databases. Various psychological and psychophysiological experiments have shown that these stimuli sets 
have great potential in the investigation of sadness, fear, and disgust emotion (Baumgartner et al. 2006; Brown et al. 2011). In addition, Mikles et al. (Mikels et al. 2005) and Redondo et al. (Redondo et al. 2008) provided a more complete characterization of the categorical structure of the IAPS and IADS stimulus set with the objective of identifying images and sounds that elicit one discrete emotion more than other emotions. The IAPS pictures ${ }^{1}$ [disgust: valence mean (SD) $=2.43(1.51)$, arousal mean $(\mathrm{SD})=5.90(2.25)$; fear: valence mean $(\mathrm{SD})=3.80(1.89)$, arousal mean $(\mathrm{SD})=5.85(2.12)$; sadness: valence mean $(\mathrm{SD})=2.74(1.57)$, arousal mean $(\mathrm{SD})=5.00$ (2.08)] and IADS sound ${ }^{2}$ [disgust: valence mean $(\mathrm{SD})=4.00(1.72)$, arousal mean $(\mathrm{SD})=5.82$ (1.93); fear: valence mean $(\mathrm{SD})=4.00(1.72)$, arousal mean $(\mathrm{SD})=5.82(1.93)$; sadness: valence mean $(\mathrm{SD})=3.28(1.65)$, arousal mean $(\mathrm{SD})=6.61(1.89)]$ were selected and combined together according to their arousal and valence values provided in the databases. For example, a negative/high aroused sound is matched with a negative/high aroused visual. Happiness, surprise, and anger emotions were elicited using video clips. A pilot study was conducted to identify the efficient video clips that would elicit the target emotion better on the participants. Ninety video clips corresponding to happiness, surprise, and anger were displayed to thirty volunteers with a mean age of 26.4 years (range: from 24 to 45 years) to rate the emotions they experienced when watching the video clips. All of the participants were psychology teachers or students at the UKM medical center, Kuala Lumpur. Of these, the thirty clips with the highest ratings were chosen for data collection. The emotion induction stimulus materials are summarized in Table 2.

${ }^{1}$ The following pictures were used for emotion induction: Disgust: 1945, 2352.2, 3000, $3010,3015,3030,3051,3060,3061,3071,3080,3110,3120,3130,3140,3150,3160,3250$, $3400,7360,7361,7380,8230,9040,9042,9181,9290,9300,9320,9330,9373,9390,9405$, 9490, 9570, 9830; Fear: 1019, 1022, 1030, 1040, 1050, 1051, 1052, 1070, 1080, 1090, 1110, 
$1111,1113,1120,1200,1201,1220,1230,1240,1280,1274,1300,1301,1302,1321,1390$, 1930, 1931, 3280, 5970, 5971, 5972, 6370, 9584, 9594, 9592; Sadness: 2205, 2271, 2276, 2490, $2520,2590,2700,2800,2900,3220,3230,3300,3301,3350,6570,6838,8010,9000,9041$, 9050, 9120, 9190, 9210, 9220, 9331, 9410, 9415, 9470, 9520, 9530, 9561,9611, 9910, 9911, 9920, 9921.

${ }^{2}$ The following sounds were used for emotion induction: Disgust: 134, 115, 251, 262, 284, 698, 702, 711, 712, 713, 714, 720, 728, 729, 730, 732, 812, 813; Fear: 106, 133, 170, 171, 275, 276, 277, 279, 291, 312, 378, 380, 424, 425, 500, 626, 627, 699, 817; Sadness: 115, 150, 260, 261, $278,280,285,286,290,293,295,310,311,368,403,420,422,501,600,625$.

\section{Emotion elicitation protocol}

An illustrated version of the emotion elicitation protocol is shown in Figure 1. As seen, the protocol had two sessions of three trials each. There was a break of $10-15$ minutes between the sessions. The participants were allowed to relax and refresh during the break, since the continuous assessment would have been too exhausting. The multimodal stimuli relating to all six emotional states (happiness, sadness, fear, anger, surprise, and disgust) were displayed in each trial in a random order. Each combination of picture and sound was presented for 6-second (Kim 2007). To maximize the participants' emotional reactivity, each clip block consisted of six combinations of the same emotional category and lasted for 36-seconds. In addition, each of the video clips varied from 36-45 seconds in duration, depending on the length of the clip. Neutral images, which can calm down the participant state, were displayed for 10 seconds at the start of each trial. This would help the participant return to the normal or neutral state from emotional excitation. A 15-second rating interval (Hamdi et al. 2012) was provided between the clips in 
which participants completed on a five point self-assessment scale. Each session lasted approximately 30 minutes.

\section{Procedure}

The purpose of the study was clearly explained to the participants before starting the experiment. The participants were further requested to relax, minimize their body movement (to reduce the appearance of relevant artifacts in the EEG recordings), and concentrate on the emotional stimuli. The self-guided emotion elicitation protocol was then displayed on the screen. The experiment set up is shown in Figure 2. At the end of each clip, participants filled out a selfassessment questionnaire to state the status of the emotions they felt during the experiment; they were also asked to report the strength of the emotions experienced using a five-point scale according to the degree $(1=$ very low, $2=$ low, $3=$ medium, $4=$ high, and $5=$ very high $)$. These ratings were used to understand the intensity of the emotional state they experienced. However, despite the intensity levels, all emotional data were taken into considerations. The participants were also allowed to indicate multiple emotions during the experiment. A picture of the selfassessment questionnaire is shown in Figure 3.

\section{Apparatus and Data analysis}

EEG recordings were measured using the Emotive EPOC 14 channel EEG wireless recording headset (Emotive Systems, Inc., San Francisco, CA) (Hadjidimitriou and Hadjileontiadis 2012). The electrode scheme was arranged according to the international 10-20 system and included active electrodes at AF3, F7, F3, FC5, T7, P7, O1, O2, P8, T8, FC6, F4, F8, and AF4 positions, referenced to the common mode sense (CMS-left mastoid)/driven right leg (DRL-right mastoid) ground. The acquired data were digitized using the embedded 16-bit ADC with $128 \mathrm{~Hz}$ sampling frequency per channel and sent to the computer via wireless technology. 
EEG analysis was performed offline in the MATLAB (version7.12.0.635, R2011a) environment. After data acquisition, the EEG signals were subjected to filtering. In particular, IIR Butterworth bandpass $\left(6^{\text {th }}\right.$ order filter) was used. The focus was placed upon the five EEG frequency bands: delta $(1-4 \mathrm{~Hz})$, theta $(4-8 \mathrm{~Hz})$, alpha $(8-13 \mathrm{~Hz})$, beta $(13-30 \mathrm{~Hz})$ and gamma $(30-49 \mathrm{~Hz})$. A study published by Kim (2007) proposed the use of different epoch sizes depending on modality (e.g., 2-6 seconds for speech, and 3-15 seconds for biosignals) (Kim 2007). In this study, the EEG signals were segmented into 6-second epochs corresponding to the duration of each multimodal stimuli projection (768 samples). A separate thresholding method was then used to remove blinking artifacts, in which epochs that were found to have amplitudes exceeding $\pm 80 \mu \mathrm{V}$ were excluded from the study (Gotlib et al. 1998). Eighty-four artifact-free epochs from the middle data segment of each emotional state were selected to compute the crosspower spectra or coherence between homologous inter-hemispheric electrode pairs.

The coherence spectrum function, $C_{x y}$, for two given signals, $x$ and $y$, is defined as, $C_{x y}(f)=\left|P_{x y}(f)\right|^{2} /\left(P_{x x}(f) \cdot P_{y y}(f)\right)$, with $\mathrm{f}$ denoting frequency, and $P$ power or cross spectrum. $\left|P_{x y}(f)\right|$ is the cross-spectrum between signals $x$ and $y$, while $P_{x x}(f)$ and $P_{y y}(f)$ are the autospectrum of the signals $x$ and $y$, respectively (Guevara and Corsi-Cabrera 1996). In this study, inter-hemispheric EEG coherence was calculated between the seven homologous electrode pairs: anterior frontal (AF3-AF4), lateral frontal (F7-F8), mid-frontal (F3-F4), fronto-central (FC5FC6), temporal (T7-T8), parietal (P7-P8), and occipital (O1-O2) (Figure 4). The EEG coherence was computed using Welch's average periodogram method (Matlab's 'mscohere' function) with an NFFT of 1024 (with the resolution of $0.125 \mathrm{~Hz}$ ) and a Hanning window length of 256.

\section{Statistics}


Fisher's Z transformation was used to normalize the distribution of coherence values (Hinrichs and Machleidt 1992). The differences between the 2 groups were assessed for each frequency band (delta, theta, alpha, beta, and gamma) of EEG using a 3-way analysis of variance (ANOVA) with repeated measures, where the group (PD vs. HC) as a between-subject factor and the emotions (happiness $v s$. sadness $v s$. fear $v s$. anger $v s$. surprise $v s$. disgust) and the electrode pairs (AF3-AF4 vs. F7-F8 vs. F3-F4 vs. FC5-FC6 vs. T7-T8 vs. P7-P8 vs. O1-O2 ) as the withinsubject factors. The ANOVA interaction was tested between groups and emotions, between groups and electrode pairs, between emotions and electrode pairs and between groups, emotions, and electrode pairs. Since a significant main effect of or interaction with emotions was found for frequency bands, a post-hoc 2-way ANOVA with repeated measures was performed (where the group was the between-subject factor and the electrode pairs were the repeated factor) on all electrode pairs to assess the emotional differences. Violations of sphericity were adjusted by the Greenhouse-Geisser correction. In order to determine significance of difference for each of the electrode pair, when a significant group and electrode pair interaction was detected by ANOVA, a separate two tailed student's t-test (second post-hoc comparisons) was performed. For the results of the post-hoc 2-way ANOVA, the Bonferroni procedure was used for second post-hoc comparisons. To examine the relationships between resting EEG and the emotional response EEG, a paired t-test was performed between the coherence values for the AF3-AF4, F7-F8, F3F4, FC5-FC6, T7-T8, P7-P8, and O1-O2 electrode pairs. Furthermore, Pearson's correlation coefficient was calculated for each electrode pair. These analyses were performed for each group separately.

The data from the behavioral study (subjective ratings as well emotion recognition rates) were analyzed by separate repeated-measure ANOVAs, with group as a between-subject factor 
and emotion as a within-subject factor. Statistical significance was defined as $p<0.05$. For all analyses, the uncorrected degrees of freedom and the corrected $p$-values are reported.

\section{RESULTS}

\section{Behavioral measures}

Mean subjective ratings are shown in Table 3. Overall the ratings were higher for happiness and lower for disgust; main effect of emotion $\mathrm{F}(5,240)=7.88, p<0.0001$. No significant difference between groups and no Group $\mathrm{x}$ Emotion interaction were observed $(p>$ 0.9). In the emotion recognition task (Figure 5), performance of PD patients did not differ significantly from HC participants. Overall, happiness stimuli were recognized most (\% correct $\mathrm{M}=93.42 ; \mathrm{SD}=9.00)$, whereas disgusted faces were recognized least $[\%$ correct mean $(\mathrm{SD})=$ $69.58(3.20)], \mathrm{F}(5,240)=2.99, p=0.023$.

\section{Relationship between resting state and emotional response EEG}

Table 4 summarizes the results of the paired samples t-test and Pearson's correlations between the coherence values of 7 electrode pairs recorded in the resting state and six emotional states (happiness, sadness, fear, anger, surprise, and disgust) in PD patients and HC. Significant relationships $(p<0.05)$ were limited to theta, alpha, beta, and gamma frequency bands in all analysed electrodes. We also observed that Pearson's correlation coefficient was higher in theta, alpha, beta, and gamma frequency bands for both groups. The delta band showed a lower correlation between the coherence values of resting EEG and emotional state EEG for both the groups.

\section{Emotional EEG coherence analysis}

For the gamma band, significant differences between the factor Group $[\mathrm{F}(1,166)=48.29$, $p<0.0001]$, Emotion $[\mathrm{F}(5,830)=5.21, p=0.0284]$ and Electrode pair $[\mathrm{F}(6,996)=950.20, p<$ 
$0.0001]$ were identified. We also detected a significant Group $\mathrm{x}$ Emotion interaction $[\mathrm{F}(5,830)=$ $4.29, p=0.039]$ and Emotion $x$ Electrode pair interaction $[\mathrm{F}(30,4980)=5.11, p=0.008]$ in the alpha band. To explain Group x Emotion interaction, a post-hoc 2-way ANOVA was performed for each emotion with Group and Electrode pair. A significant interaction between these factors with sadness $[\mathrm{F}(6,996)=8.39, p<0.0001]$, fear $[\mathrm{F}(6,996)=19.20, p<0.0001]$, anger $[\mathrm{F}(6$, $996)=23.12, p<0.0001]$, and disgust $[\mathrm{F}(6,996)=28.18, p<0.0001]$ emotions was found; however, the emotions happiness and surprise did not differ in the gamma band $(p>0.05)$ (Figure 6A).

For the alpha band, a significant difference between the factor Group $[\mathrm{F}(1,166)=22.77, p$ $<0.0001]$, Emotion $[\mathrm{F}(5,830)=2.25, p=0.0472]$ and Electrode pair $[\mathrm{F}(6,996)=717.32, p<$ 0.001] were identified. Additionally, we detected a significant Group x Emotion interaction [F $(5,830)=2.27, p=0.045]$ and Emotion $\mathrm{x}$ Electrode pair interaction $[\mathrm{F}(30,4980)=2.11, p=$ 0.005] in the alpha band. To explain the Group x Emotion interaction, a post-hoc 2-way ANOVA was performed for each emotion with Group and Electrode pairs. A significant interaction between these factors with sadness $[\mathrm{F}(6,996)=6.53, p<0.0001]$, fear $[\mathrm{F}(6,996)=13.05, p<$ $0.0001]$, anger $[\mathrm{F}(6,996)=14.65, p<0.0001]$, and disgust $[\mathrm{F}(6,996)=10.29, p<0.0001]$ emotions was identified. The emotions happiness and surprise did not differ in the alpha band ( $p$ $>0.05)$ (Figure 6B).

PD patients differed significantly from controls across the theta band $[\mathrm{F}(1,166)=27.66, p$ $<0.0001]$. Significant differences were also found between the factor Emotions $[F(5,830)=$ 2.34, $p=0.0445]$ and Electrode pair $[\mathrm{F}(6,996)=819.95, p<0.0001]$. We also detected a significant interaction between Group $\mathrm{x}$ Emotion $[\mathrm{F}(5,830)=2.47, p=0.0311]$ and Emotion $\mathrm{x}$ Electrode pair $[\mathrm{F}(30,4980)=2.41, p=0.001]$ factor. This interaction can be explained by the 
separate post-hoc 2-way comparisons with Group and Electrode pair for each emotion. We found a significant interaction between these factors with the emotions sadness $[\mathrm{F}(6,996)=6.53, p<$ $0.0001]$, fear $[\mathrm{F}(6,996)=13.05, p<0.0001]$, anger $[\mathrm{F}(6,996)=14.65, p<0.0001]$, and disgust $[\mathrm{F}(6,996)=10.29, p<0.0001]$ in the theta band (Figure 6C). The coherence values for the emotions happiness and surprise were not significant between the groups $(p>0.6)$.

There was no significant Group and Electrode pair main effect difference detected across the beta band $(p>0.05)$; however, we detected a significant Emotion $[\mathrm{F}(5,830)=2.50, p=$ 0.0292] and Group $x$ Emotion interaction effect $[F(5,830)=2.29, p=0.0438]$ in this band. To explain this interaction, we performed separate 2-way ANOVA for each emotion within the Group and Electrode pair. PD patients showed significantly lower inter-hemispheric coherences in the emotions sadness $[\mathrm{F}(6,996)=6.00, p<0.0001]$, fear $[\mathrm{F}(6,996)=7.89, p<0.0001]$, anger $[\mathrm{F}(6,996)=9.10, p<0.0001]$, and disgust $[\mathrm{F}(6,996)=10.53, p<0.0001]$ than $\mathrm{HC}$ participants (Figure 6D). No differences were detected for the emotions happiness and surprise across the beta band $(p>0.05)$.

Finally, the delta band of the PD and the control groups did not differ in inter-hemispheric coherences during emotion processing $(p>0.3)$ (Figure 6E).

Table 5 summarizes the results of the post-hoc t-test analysis between the coherence values of each electrode pairs in PD patients and $\mathrm{HC}$ during emotion processing. The electrode pair significance was calculated for the frequency bands that significantly separated the PD patient groups and HC (theta, alpha, beta, and gamma). Overall PD patients showed decreased inter-hemispheric coherence values compared to $\mathrm{HC}$ participants for all of the significant electrode pairs during emotion processing. Figure 7 shows the inter-hemispheric coherence spectra averaged across seven electrode pairs of PD patients and $\mathrm{HC}$ during emotion processing. 
It can be clearly found that coherences in all the seven electrode pairs for PD patients were lower compared to $\mathrm{HC}$, suggesting a pattern of under-connectivity in PD patients during emotion information processing.

\section{DISCUSSION}

EEG coherence is a frequency specific measure of the degree of pairing between spatially separated brain electrode locations (French and Beaumont 1984). Since coherence is derived from correlation measures, it is usually interpreted as a reflection of functional and structural connectivity (Guevara and Corsi-Cabrera 1996). The current study investigated behavioral measures (subjective ratings and emotion recognition accuracy) and inter-hemispheric EEG coherence in response to six emotional states (happiness, sadness, fear, anger, surprise, and disgust) of PD patients compared to $\mathrm{HC}$ participants, and focused on establishing whether there were any EEG coherence changes associated with emotion information processing, and whether coherence analysis could be used as a biomarker of emotional impairments associated with PD patients. Seven electrode pairs (AF3-AF4, F7-F8, F3-F4, FC5-FC6, T7-T8, P7-P8, and O1-O2) were selected to represent the seven brain regions, which are the anterior frontal, lateral frontal, mid-frontal, fronto-central, temporal, parietal, and occipital regions.

In line with previous findings on intact emotion recognition in PD (Adolphs et al. 1998; Dujardin et al. 2004; Pell and Leonard 2005; Wieser et al. 2012), the present study also found that PD patients showed no impairments in emotion recognition accuracy and subjective ratings during viewing of the emotional stimuli. It is noteworthy that these findings are most likely not due to low statistical power, since PD patients were descriptively even better in recognizing the emotional stimuli for happiness, fear, and disgust compared to HC participants (see Figure 5). More recently, it was found that PD patients under dopamine replacement therapy (DRT) were 
not impaired in emotion recognition, but more sensitive to cognitive load (Cohen et al. 2010), which seems to be especially true for non-demented and non-depressive samples like in our study. In accordance with the EEG data, coherence measures at theta, alpha, beta, and gamma frequency bands were significantly different between PD patients and HC during emotion processing. The results suggest that there may be neuropathological evidence indicating that PD is associated with slowing of inter-hemispheric EEG synchronization (Neufeld et al. 1988; Yeager et al. 1966), which had a significant correlation with progression of the Hoehn and Yahr Stage in PD (Morita et al. 2009). There was no significant difference between delta band coherence during emotion processing, which was mostly likely due to the fact that the patients were predominantly in the mild stage of PD. Furthermore, PD patients showed decreased coherence at delta, theta, alpha, beta, and gamma frequency bands compared to $\mathrm{HC}$, suggesting that functional disconnections exist between cortical areas, which could be interpreted as a neocortical "disconnection syndrome" for PD (Cronin-Golomb 2010). In contrast, previous EEG studies (Wieser et al. 2012; Wieser et al. 2006) reported that there was no impairment in emotion processing in PD patients as reflected in behavioral and neurophysiological measures. The discrepancy in the current results may have been influenced by many factors, such as stimuli sets, disease duration, medical treatment, illness severity, and motor disability (Gray and TickleDegnen 2010; Yuvaraj et al. 2013). It has already been shown that impairments in emotion processing in PD may vary as a function of stimulus modality-visual or auditory (Lima et al. 2013)-but this neurophysiological dissociation using a multimodal approach is a new finding. The PD patients in the present study had quite a broad range of disease duration (1-12 years), a great variety of medical treatments, and the range of Hoehn and Yahr scores was relatively wide (range from 1-3). Because of these heterogeneities clinical profiles of our sample, our findings 
must be interpreted cautiously. In particular, medication status and illness severity may have influenced the subjective ratings and neurophysiologic measures.

Interestingly, our study also showed that inter-hemispheric coherence for negative emotions (sadness, fear, anger, and disgust) was significantly lower in the PD patients than the HC participants in theta, alpha, beta, and gamma frequency bands; however, no significant difference was found in positive emotions (happiness and surprise), which is also in line with the results of other studies indicating that PD patients were more impaired in recognizing negative emotions than relatively positive emotions (Gray and Tickle-Degnen 2010; Péron et al. 2012; Dujardin et al. 2004; Sprengelmeyer et al. 2003; Suzuki et al. 2006). Many researchers have suggested that individuals with PD may be particularly impaired in recognizing negative emotions because of dysfunctions in specific emotion-related brain areas. Lawrence et al. (2007) reviewed evidence suggesting a central role of ventral striatal dopamine systems in anger recognition (Lawrence et al. 2007). The authors suggested that dysfunction in these systems explains their finding of a selective impairment in anger emotion during acute withdrawal from DRT. Suzuki et al. (2006) showed that the basal ganglia and the insula are involved in the recognition of disgust; both regions are dysfunctional in PD (Suzuki et al. 2006). In addition, other studies have shown inconsistent impairments in the recognition of disgust (Kan et al. 2004; Sprengelmeyer et al. 2003) as well as in the recognition of fear (Ariatti et al. 2008; Sprengelmeyer et al. 2003). It is widely accepted that the amygdala is involved in the recognition of fear emotion (Adolphs et al. 1996) and there is evidence that the amygdala is affected in PD patients (Bouchard et al. 2008; Tessitore et al. 2002). Finally, the left amygdala also appears to play a role in the recognition of the sadness emotion (Blair et al. 1999). Apart from the specific neural circuits' dysfunction hypothesis, the most widely believed hypothesis is 
dopamine depletion. There is a large body of evidence pointing to the involvement of dopamine in emotion processing (Salgado-Pineda et al. 2005). For example, the association between dopaminergic activity and emotion processing has been demonstrated in healthy male volunteers that received a dopamine D2-antagonist, which caused impaired recognition of angry faces (Lawrence et al. 2002). Using PET, it has been shown that reduced dopamine transporter availability (DAT) is related to a decrease in the activation of emotional gesture recognition (Lotze et al. 2009). However, our PD patients were taking dopaminergic medication during the study and still revealed signs of dopamine deficiency, as indicated by a mean value of 17.05 in the motor part of the UPDRS. This finding is in line with previous studies demonstrating that the difficulties that PD patients processing emotional information's are only partly improved by dopaminergic therapy (Tessitore et al. 2002). Thus, dopamine depletion could explain the difficulties in processing emotional information, although involvement of other transmitter systems such as the serotoninergic or noradrenergic cannot be excluded.

Some limitations of this study should be considered. Since the evaluated electrodes are relatively close to each other, it is important to rule out potential effects of volume conduction. Also, the use of a small number of PD samples, which can affect the reliability. Taken together, further research may need to avoid the volume conduction artifact by using an imaginary part of $\underline{\text { coherency (Nolte et al. 2004) with larger number of PD samples. This could build a more }}$ understanding of relationship between brain functional connectivity and emotion. In addition, the PD patients in this study were considered of both affected sides of the body (predominantly leftsided and right-sided motor symptoms). The distinction between predominantly affected sides in PD individuals should be considered in future PD studies as these subgroup differences within PD might provide hopeful leads for intervention and rehabilitation. Moreover, all PD patients 
were under DRT, which might have also affected their performance in emotion processing (Tessitore et al. 2002). Future studies should be conducted with unmedicated patients to reveal the pure effect of PD. Moreover, differences between intra-hemispheric functional connectivity as a measure of investigating different brain regions for emotion processing were not explored. In summary, this is one of the first studies to combine EEG coherence measurements with an emotion processing and subjective rating procedure to investigate possible impairments in emotion processing in PD. No impairments in behavioral measures were found, but decreased coherence reactivity to emotional stimuli was revealed by EEG measures in PD patients. These results could indicate a precursor of a stronger emotion deficit, which is already present in patients with mild PD receiving DRT. Controlled studies with PD patients on and off medication could help to clarify the influence of dopaminergic medication on emotion processing in PD. 


\section{ACKNOWLEDGEMENTS}

The research was financially supported by Ministry of Science and Technology (MOSTI), Malaysia. Grant Number: 9005-00053. The authors would like to thank Dr. Mohamad Fadli, Dr. Siva Rao Subramanian and Dr. Shahrul Azmin for their assistance with recruitment of PD participants. Also we would like to thank all of the individuals who participated in this study. 


\section{REFERENCES}

Adolphs R, Damasio H, Tranel D, Damasio AR (1996) Corticle systems for the recognition of emotion in facial expressions J Neurosci 16:7678-7687

Adolphs R, Schul R, Tranel D (1998) Intact recognition of facial emotion in Parkinson's disease. Neuropsychology 12 (2):253-258

Ariatti A, Benuzzi F, Nichelli P (2008) Recognition of emotions from visual and prosodic cues in Parkinson's disease. Neurol Sci 29 (4):219-227

Bastiaanse R, Leenders KL (2009) Language and Parkinson's disease. Cortex 45 (8):912-914

Baumgartner T, Esslen M, Jancke L (2006) From emotion perception to emotion experience: Emotions evoked by pictures and classical music. Int J Psychophysiol 60 (1):34-43

Beck AT, Ward CH, Mendelson M, Mock J, Erbaugh J (1961) An inventory for measuring depression. Arch of Gen Psychiatry 4:561-571

Beyer MK, Janvin CC, Larsen JP, Aarsland D (2007) A magnetic resonance imaging study of patients with Parkinson's disease with mild cognitive impairment and dementia using voxel-based morphometry. J Neurol Neurosurg Psychiatry 78 (3):254-259

Blair RJR, Morris JS, Frith CD, Perrett DI, Dolan RJ (1999) Dissociable neural responses to facial expressions of sadness and anger. Brain 122:883-893

Bouchard TP, Malykhin N, Martin WR, Hanstock CC, Emery DJ, Fisher NJ, Camicioli RM (2008) Age and dementia-associated atrophy predominates in the hippocampal head and amygdala in Parkinson's disease. Neurobiol Aging 29 (7):1027-1039

Bowers D, Miller K, Mikos A, Kirsch-Darrow L, Springer U, Fernandez H, Foote K, Okun M (2006) Startling facts about emotion in Parkinson's disease: blunted reactivity to aversive stimuli. Brain 129 (Pt 12):3356-3365 
Bradley MM, Lang PJ (2007) International affective digitized sounds (2nd Edition; IADS-2):

Affective ratings of sounds and instruction manual. Technical Report B-3 University of Florida, Gainesville, FL

Brown L, Grundlehner B, Penders J (2011) Towards wireless emotional valence detection from EEG. IEEE Engineering in Medicine and Biology Society: 2188-2191

Busk J, Galbraith GC (1975) EEG correlates of visual-motor practice in man. Electroencephalogr Clin Neurophysiol 38:415-422

Caekebeke JF, Jennekens-Schinkel A, VanderLinden ME, Buruma OJ, Roos RA (1991) The interpretation of dysprosody in patients with Parkinson's disease. J Neurol Neurosurg \& Psychiatry 54 (2):145-148

Cancelliere AEB, Kertesz A (1990) Lesion Localization in Acquired Deficits of Emotional Expression and Comprehension. Brain Cogn 13 (2):133-147

Celesia GG, Wanamaker WM (1972) Psychiatric disturbances in Parkinson's disease. Dis Nerv Syst 33 (9):577-583

Chen HL, Huang CC, Yu XG, Xu X, Sun X, Wang G, Wang SJ (2013) An efficient diagnosis system for detection of Parkinson's disease using fuzzy k-nearest neighbor approach. Expert Syst Appl 40 (1):263-271

Clark US, Neargarder S, Cronin-Golomb A (2008) Specific Impairments in the Recognition of Emotional Facial Expressions in Parkinson's disease. Neuropsychologia 46 (9):23002309

Cohen H, Gagne M-H, Hess U, Pourcher E (2010) Emotion and object processing in Parkinson's disease. Brain Cogn 72:457-463 
Cronin-Golomb A (2010) Parkinson's disease as a Disconnection Syndrome. Neuropsychol Rev $20(2): 191-208$

Dara C, Monetta L, Pell MD (2008) Vocal emotion processing in Parkinson's disease: Reduced sensitivity to negative emotions. Brain Res 1188:100-111

Davidson RJ (1992) Anterior cerebral asymmetry and the nature of emotion. Brain Cogn 20

$$
\text { (1):125-151 }
$$

Dujardin K, Blairy S, Defebvre L, Duhem S, Noël Y, Hess U, Destée A (2004) Deficits in decoding emotional facial expressions in Parkinson's disease. Neuropsychologia 42 (2):239-250

Fahn S, Elton RL, Committee M (1987) Unified Parkinson's disease Rating Scale. In: Fahn's, Marsden CD, Calne DB, Goldstein M, Clane DB Recent Developments in Parkinson's Disease Macmillan Health Care Information; Florham Park: 2:153-163

Folstein MF, Folstein SE, Mchugh PR (1975) Mini-Mental State Examination: a practical method for grading the cognitive state of patients. Psychol Res 12 (3):189-198

Fonseca LC, Tedrus GMAS, Carvas PN, Machado ECFA (2013) Comparision of quantitative EEG between patients with Alzheimer's disease and those with Parkinson's disease dementia. Clin Neurophysiol 124:1970-1974

French CC, Beaumont JC (1984) A critical review of EEG coherence studies of hemisphere function. Int J Psychophysiol 1:241-254

Garrido-Vásquez P, Pell MD, Paulmann S, Strecker K, Schwarz J, Kotz SA (2012) An ERP study of vocal emotion processing in asymmetric Parkinson's disease. Soc Cogn Affect Neurosci 8(8):918-27 
Gasser T, Jennen-Steinmetz C, Verleger R (1987) EEG coherence at rest and during a visual task in two groups of children. Electroencephalogr Clin Neurophysiol 67: 151-158

Gotlib IH, Raganathan C, Rosenfeld JP (1998) Frontal EEG alpha asymmetry, depression, and cognitive functioning. Cogn Emo 12 (3):449-478

Gray HM, Tickle-Degnen L (2010) A meta-analysis of performance on emotion recognition tasks in Parkinson's disease. Neuropsychology 24 (2):176-191

Gross JJ, Levenson RW (1995) Emotion elicitation using films. Cogn Emo 9:87-108

Guevara MA, Corsi-Cabrera M (1996) EEG coherence or EEG correlation?. Int J Psychophysiol $23(3): 145-153$

Hadjidimitriou SK, Hadjileontiadis LJ (2012) Toward an EEG-based recognition of music liking using time-frequency analysis. IEEE Trans Biomed Eng 59 (12):3498-3510

Hamdi H, Richard P, Suteau A, Allain P (2012) Emotion assessment for affective computing based on physiological responses. IEEE proceedings of World Congress on Computational Intelligence: 10-15

Hinrichs H, Machleidt W (1992) Basic emotions reflected in EEG-coherences. Int J Psychophysiol 13 (3):225-232

Hoehn MM, Yahr MD (1967) Parkinsonism: Onset, Progression and mortality. Neurology 17 (5):427-442

Jerritta S, M Murugappan, Wan K, Yaacob S (2013) Electrocardiogram-based emotion recognition system using empirical mode decomposition and discrete Fourier transform. Expert Syst. doi: 10.1111/exsy.12014 
Kan Y, Mimura M, Kamijima K, Kawamura M (2004) Recognition of emotion from moving facial and prosodic stimuli in depressed patients. J Neurol Neurosurg Psychiatry 75:16671671

Kim J (2007) Bimodal emotion recognition using speech and physiological changes. Technical Report

Kim KH, Bang SW, Kim SR (2004) Emotion recognition system using short-term monitoring of physiological signal. Med Biol Eng Comput 42:419-427

Kober H, Barrett LF, Joseph J, Bliss-Moreau E, Lindquist K, Wagera TD (2008) Functional grouping and cortical-subcortical interactions in emotion: A meta-analysis of neuroimaging studies. Neuroimage 42 (2):998-1031

Lang PJ, Greenwald MK, Bradley MM, Hamm AO (1993) Looking at the pictures: affective, facial, visceral, and behavioral reactions. Psychophysiology 30 (3):261-273

Lawrence AD, Calder AJ, McGowan SW, Grasby PM (2002) Selective disruption of the recognition of facial expressions of anger. Neuroreport 13 (6):881-884

Lawrence AD, Goerendt IK, Brooks DJ (2007) Impaired recognition of facial expression of anger in Parkinson's disease patients acutely withdrawn from dopamine replacement therapy. Neuropsychologia 45 (1):65-74

Leuchter AF, Newton TF, Cook IA, Walter DO, Rosenberg-Thompson S, Lachenbruch PA (1992) Changes in brain functional connectivity in Alzheimer-type and multi-infarct dementia. Brain 115 (5):1543-1561

Levin BE, Llabre MM, Weiner WJ (1989) Cognitive impairments associated with early Parkinson's disease. Neurology 39 (4):557-561 
Lima CF, Garrett C, Castro SL (2013) Not all the sounds sound the same: Parkinson's disease affects differently emotion processing in music and in speech prosody. J Clin Exp Neuropsychol 35 (4):373-392

Lotze M, Reimold M, Heymans U, Laihinen A, Patt M, Halsband U (2009) Reduced ventrolateral fMRI response during observation of emotional gestures related to the degree of dopaminergic impairment in Parkinson disease. J Cogn Neurosci 21 (7):13211331

Madeley P, Ellis A, Mindham R (1995) Facial expressions and Parkinson's disease. Behavioral Neurology 8 (2):115-119

Mikels J, Fredrickson B, Larkin G, Lindberg C, Maglio S, Reuter-Lorenz P (2005) Emotional Category data on images from the international affective picture system. Behav Res Methods 37 (4):630-636

Miller KM, Okun MS, Marsiske M, Fennell EB, Bowers D (2009) Startle reflex hyporeactivity in Parkinson's disease: an emotion-specific or arousal-modulated deficit? Neuropsychologia 47:1917-1927

Mohr E, Juncos J, Cox C, Litvan I, Fedio P, Chase TN (1990) Selective deficits in cognition and memory in high-functioning Parkinsonian patients. J Neurol Neurosurg Psychiatry 53 (7):603-606

Morita A, Kamei S, Serizawa K, Mizutani T (2009) The Relationship Between Slowing EEGs and the Progression of Parkinson's disease. J Clin Neuropsychol 26 (6):426-429

Murugappan M, Rizon M, Nagarajan R, Yaacob S (2009) An Investigation on Visual and Audiovisual Stimulus based Human Emotion Recognition using EEG. Int J Med Eng Informat 1 (3): 342-356 
Neufeld MY, Inzelberg R, Korczyn AD (1988) EEG in demented and non-demented parkinsonian patients. Acta Neurol Scand 78 (1):1-5

Newton TF, Leuchter AF, Walter DO, Gorp WGV, Morgenstern H, Miller EN, Lieb K, Visscher B, Satz P, Weiner H (1994) Electroencephalographic coherence in acquired immune deficiency syndrome. Psychiatric Res 54 (1):1-11

Nolte G, Bai O, Wheaton L, Mari Z, Vorbach S, Hallett M (2004) Identifying true brain interaction from EEG data using the imaginary part of coherency. Clin Neurophysiol $115: 2292-2307$

Obeso JA, Marin C, Rodriguez-Oroz C, Blesa J, Benitez-Temino B, Mena-Segovia J, Rodríguez M, Olanow CW (2008) The basal ganglia in Parkinson's disease: current concepts and unexplained observations. Ann Neurol 64:S30-S46

Oldfield RC (1971) The assessment and analysis of handedness: the Edinburgh inventory. Neuropsychologia 9 (1):97-113

Paulmann S, Pell MD (2010) Dynamic emotion processing in Parkinson's disease as a function of channel availability. J Clin Exp Neuropsychol 32 (8):822-835

Pell MD, Leonard CL (2003) Processing emotional tone from speech in Parkinson's disease: A role for the basal ganglia. Cogn Affect Behav Neurosci 3 (4):275-288

Pell MD, Leonard CL (2005) Facial expression decoding in early Parkinson's disease. Cogn Brain Res 23 (3):327-340

Péron J, Dondaine T, Jeune FL, Grandjean D, Vérin M (2012) Emotional processing in Parkinson's disease: a systematic review. Mov Disord 27 (2):186-199 
Petrantonakis PC, Hadjileontiadis LJ (2011) A novel emotion elicitation index using frontal brain asymmetry for enhanced EEG-based emotion. IEEE Trans Inf Technol Biomed 15 (5):737-746

Pillon B, Dubois B, Agid Y (1996) Testing cognition may contribute to the diagnosis of movement disorders. Neurology 46 (2):329-334

Redondo J, Fraga I, Padron I, Pineiro A (2008) Affective ratings of sound stimuli. Behav Res Methods 40 (3):784-790

Roemer RA, Shagass C, Dubin W, Jaffe R, Siegal L (1992) Quantitative EEG in elderly depressives. Brain Topogr 4 (4):285-290

Salgado-Pineda P, Delaveau P, Blin O, Nieoullon A (2005) Dopaminergic contribution to the regulation of emotional perception. Clin Neuropharmacol 28 (5):228-237

Savitt JM, Dawson VL, Dawson TM (2006) Diagnosis and treatment of Parkinson disease: molecules to medicine. The Journal of Clinical Investigation 116 (7):1744-1754

Schellberg D, Besthorn C, Klos T, Gasser T (1990) EEG power and coherence while male adults watch emotional video films. Int J Psychophysiol 9:279-291

Schröder C, Möbes J, Schütze M, Szymanowski F, Nager W, Bangert M, Munte TF, Dengler R (2006) Perception of emotional speech in Parkinson's disease. Mov Disord 21 (10):17741778

Sergerie K, Chochol C, Armony JL (2008) The role of the amygdala in emotional processing: A quantitative meta-analysis of functional neuroimaging studies. Neurosci Biobehav Rev $31: 811-830$

Shaw JC, O'Connor P, ongley C (1977) The EEG as a measure of cerebral functional organization. Br J Psychiatry 130:260-264 
Soikkeli R, Partanen J, Soininen H, Paakkonen A, Riekkinen PS (1991) Slowing of EEG in Parkinson's disease. Electroencephalogr Clin Neurophysiol 79 (3):159-165

Sprengelmeyer R, Young AW, Mahn K, Schroeder U, Woitalla D, Büttner T, Kuhn W, Przuntek H (2003) Facial expression recognition in people with medicated and unmedicated Parkinson's disease. Neuropsychologia 41 (8):1047-1057

Suzuki A, Hoshino T, Shigemasu K, Kawamura M (2006) Disgust-specific impairment of facial expression recognition in Parkinson's disease. Brain 129 (3):707-717

Tessitore A, Hariri A, Fera F, Smith W, Chase T, Hyde T, Weinberger D, Mattay V (2002) Dopamine modulates the response of the human amygdala: A Study in Parkinson's disease. J Neurosci 22 (20):9099-9103

Thatcher RW, Walker RA, Gerson I, Geisler FH (1989) EEG discriminant analyses of mild head trauma. Electroencephalogr Clin Neurophysiol 73 (2):94-106

Ventura MI, Baynes K, Sigvardt KA, Unruh AM, Acklin S, Kirsch HE, Disbrow EA (2012) Hemispheric asymmetries and prosodic emotion recognition deficits in Parkinson's disease Neuropsychologia 50:1936-1945

Wang Y, Guan L (2008) Recognizing human emotional state from audiovisual signals. IEEE Trans Multimed 10 (4):659-668

Wieser MJ, Klupp E, Weyers P, Pauli P, Weise D, Zeller D, Classen J, Muhlberger A (2012) Reduced early visual emotion discrimination as an index of diminished emotion processing in Parkinson's disease? - Evidence from event-related brain potentials. Cortex 48 (9):1207-1217 
Wieser MJ, Muhlberger A, Alpers G, Macht M, Ellgring H, Pauli P (2006) Emotion processing in parkinson's disease: Dissociation between early neuronal processing and explicit ratings. Clin Neurophysiol 117 (1):94-102

Yeager CL, Alberts WW, Denature LD (1966) Effect of stereotaxic surgery upon electroencephalographic status of parkinsonian patients. Neurology 16:904-910

Yip JT, Lee TM, Ho SL, Tsang KL, Li LS (2003) Emotion recognition in patients with idiopathic Parkinson's disease. Mov Disord 18 (10):1115-1122

Yuvaraj R, Murugappan M, Norlinah MI, Sundaraj K, Khairiyah M (2013) Review of Emotion Recognition in Stroke Patients. Dement Geriatr Cogn Disord 36:179-196 
1

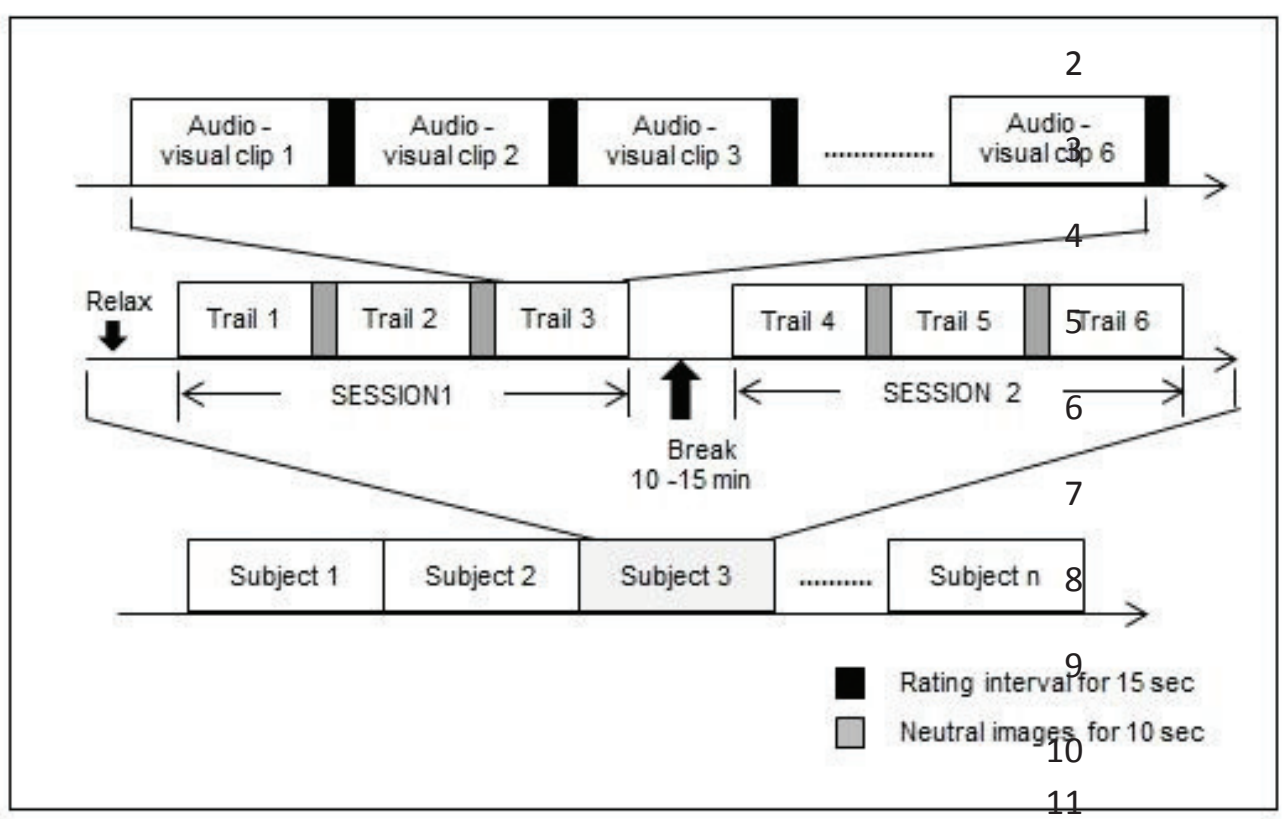

Fig. 1 Emotion elicitation protocol

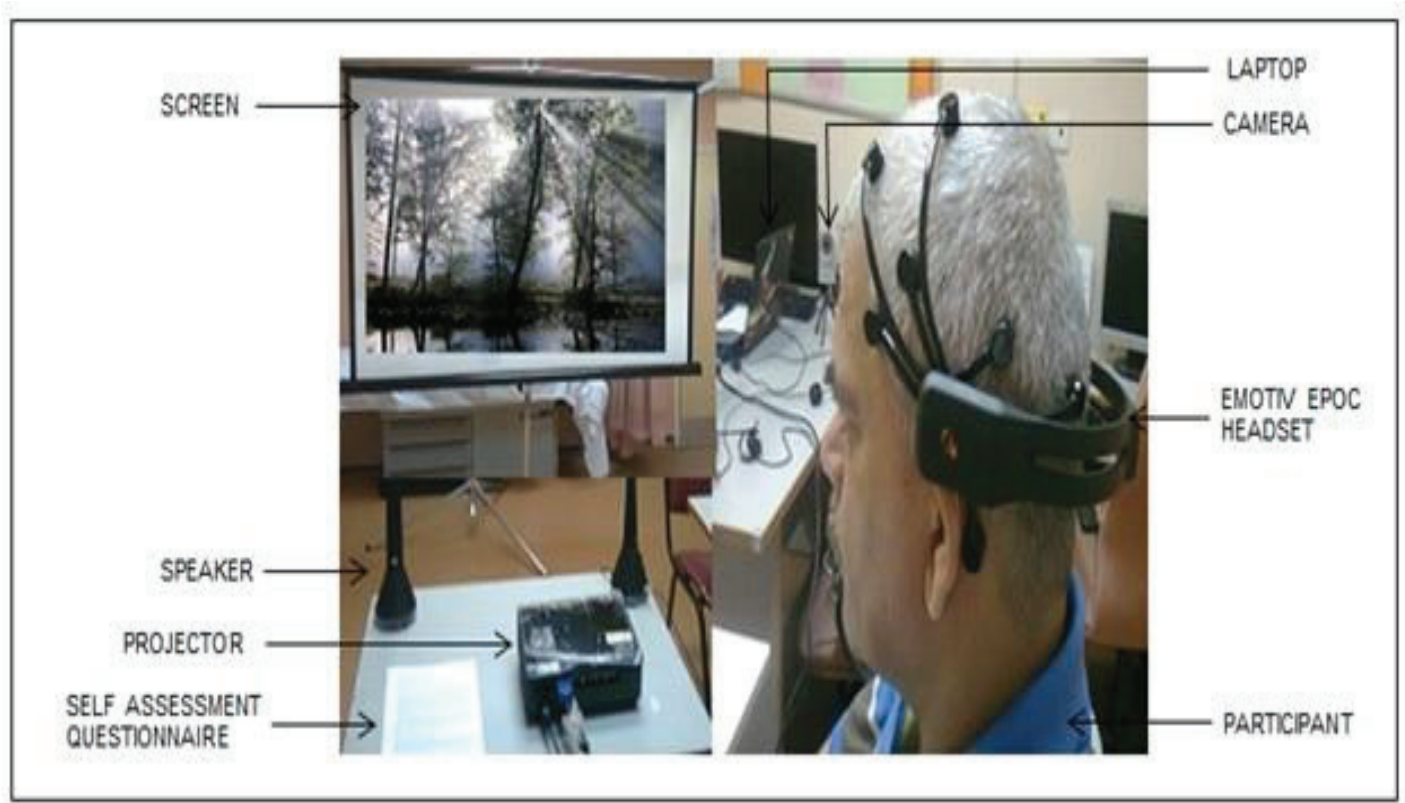

Fig. 2 Experimental setup 


\begin{tabular}{|c|c|c|c|c|c|c|c|c|c|c|c|c|c|c|c|c|c|c|}
\hline \multirow{2}{*}{\begin{tabular}{|c} 
S. \\
NO
\end{tabular}} & \multirow{2}{*}{$\begin{array}{l}\text { Audio- } \\
\text { visual } \\
\text { clip No. }\end{array}$} & \multicolumn{6}{|c|}{$\begin{array}{l}\text { Primary emotion experienced } \\
\text { (V) }\end{array}$} & \multicolumn{5}{|c|}{$\begin{array}{c}\text { Intensity of primary emotion } \\
\text { (V) }\end{array}$} & \multicolumn{6}{|c|}{$\begin{array}{c}\text { Any other emotion? } \\
\text { (V) }\end{array}$} \\
\hline & & HAPPY & SAD & FEAR & ANGEF & SURPRISE & DISGUST & $\begin{array}{l}\text { VERY } \\
\text { LOW }\end{array}$ & LOW & MEDIUN & HIGH & $\begin{array}{l}\text { VERY } \\
\text { HIGH }\end{array}$ & HAPPY & SAD & FEAR & ANGER & SURPRISE & DISGUST \\
\hline 1 & Clip 1 & & & & & & & & & & & & & & & & & \\
\hline 2 & Clip 2 & & & & & & & & & & & & & & & & & \\
\hline 3 & Clip 3 & & & & & & & & & & & & & & & & & \\
\hline 4 & Clip 4 & & & & & & & & & & & & & & & & & \\
\hline 5 & Clip 5 & & & & & & & & & & & & & & & & & \\
\hline 6 & Clip 6 & & & & & & & & & & & & & & & & & \\
\hline 7 & Clip 7 & & & & & & & & & & & & & & & & & \\
\hline 8 & Clip 8 & & & & & & & & & & & & & & & & & \\
\hline 9 & Clip 9 & & & & & & & & & & & & & & & & & \\
\hline 10 & Clip 10 & & & & & & & & & & & & & & & & & \\
\hline 11 & Clip 11 & & & & & & & & & & & & & & & & & \\
\hline
\end{tabular}

Fig. 3 Picture of self-assessment questionnaire

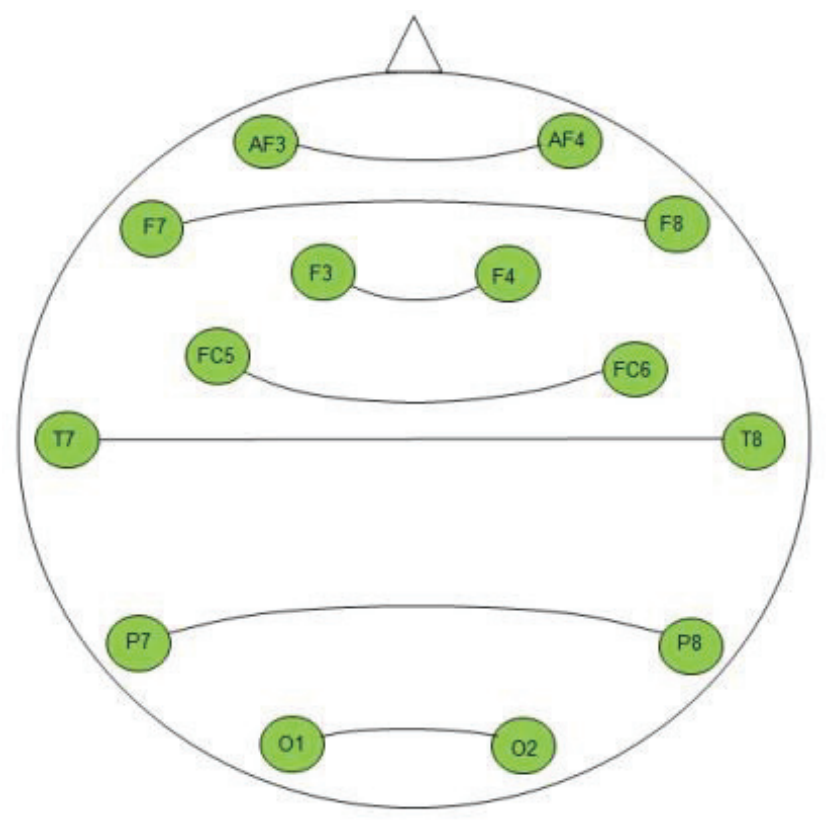

Fig. 4 Homologous inter-hemispheric electrode pairs 


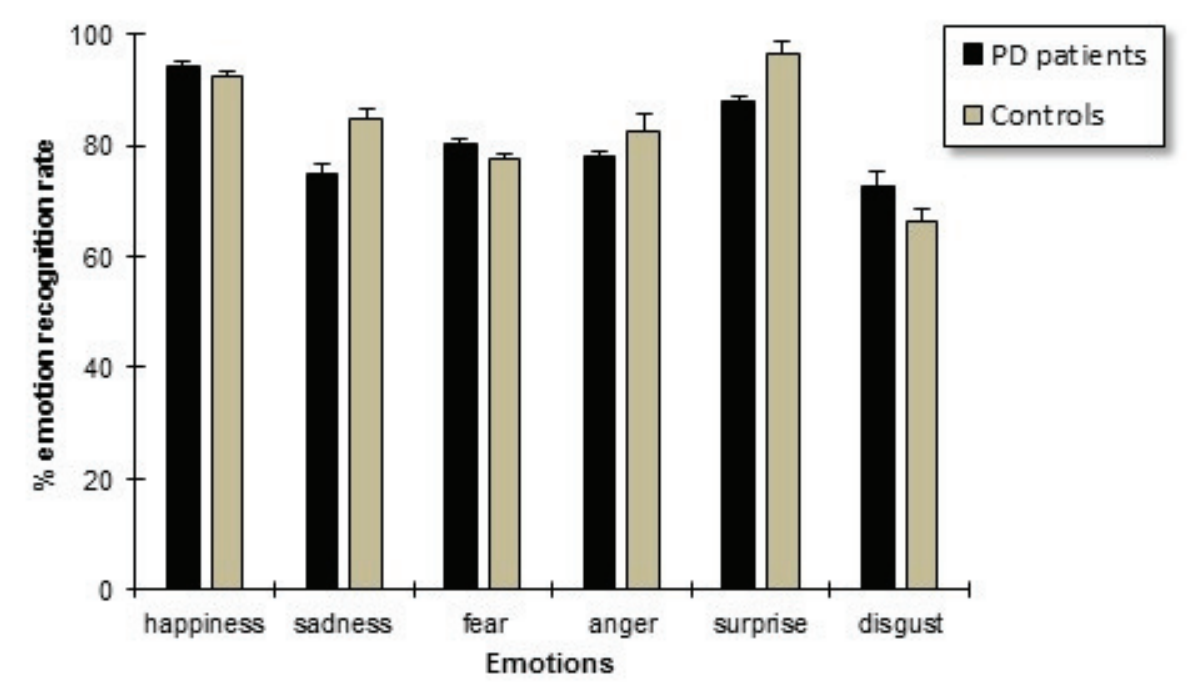

Fig. 5 Means and standard error of means (SEM) of emotion recognition accuracy (\%) in PD and HC groups 

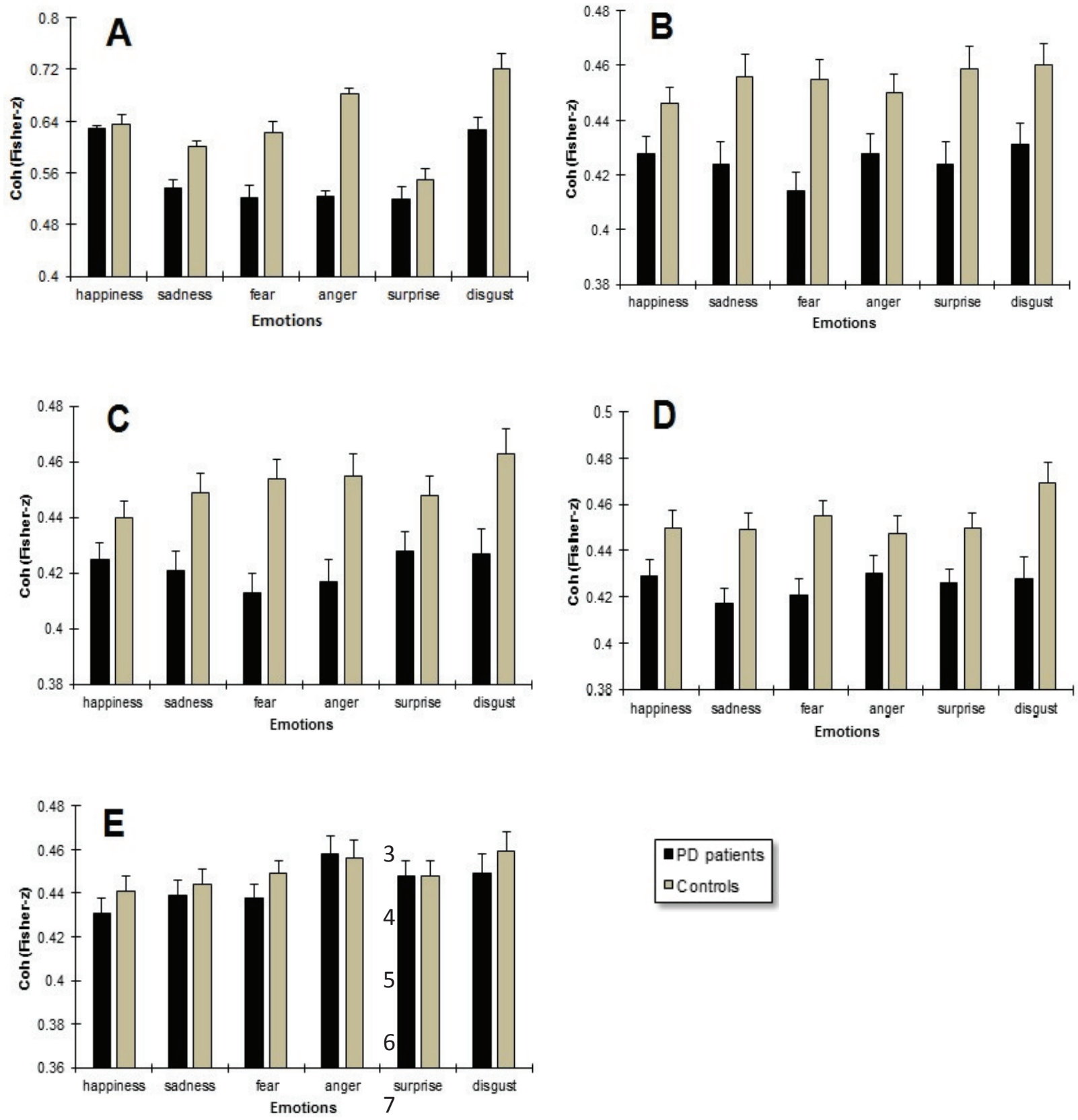

-PD patients

口Controls

Fig. 6 Inter-hemispheric coherence values of six emotions in PD and HC groups. (A), Gamma band coherence $(30-49 \mathrm{~Hz})$; (B) Alpha band coherence $(8-13 \mathrm{~Hz})$; (C) Theta band coherence (4 -8 Hz); (D) Beta band coherence $(13-30 \mathrm{~Hz})$; (E) Delta band coherence (4 - $8 \mathrm{~Hz})$; Values are mean \pm SEM of coherence values transformed to Fisher's Z scores. coh, coherence 

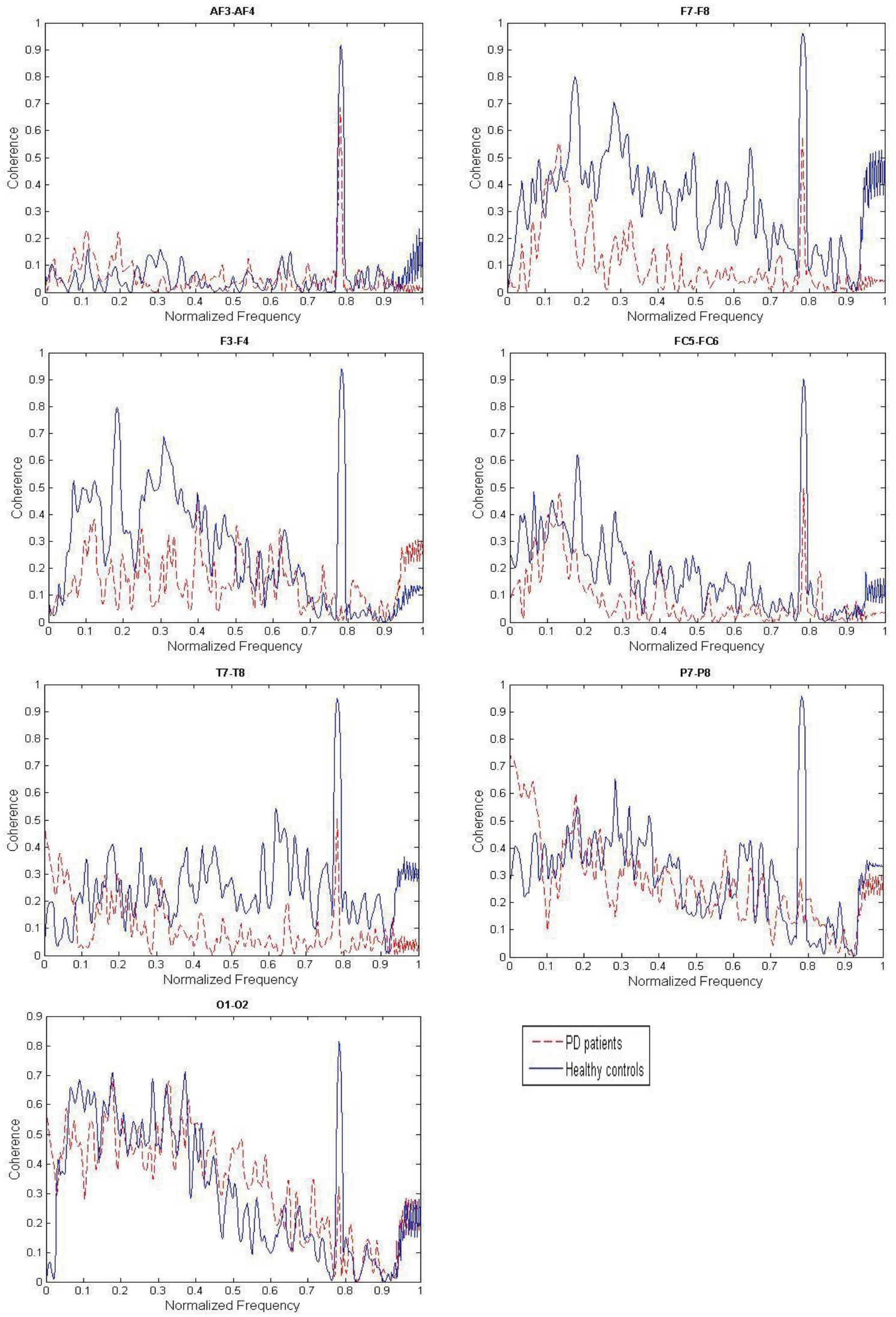
Fig.7 Coherence spectra averaged across all the seven electrode pairs between PD patients and healthy controls during emotion processing. 
Table 1 Demographic and clinical characteristics (mean and SDs) of PD patients and healthy controls

\begin{tabular}{ccccc}
\hline & PD $(\mathbf{N}=\mathbf{2 0})$ & HC $(\mathbf{N}=\mathbf{3 0})$ & Test's & Statistical \\
& & & Value & result* \\
\hline Age (years) & $59.05(5.64)$ & $58.43(3.01)$ & $\mathrm{t}=0.502$ & $p=0.618$ \\
\hline Gender & $10 \mathrm{~F} / 10 \mathrm{M}$ & $17 \mathrm{~F} / 13 \mathrm{M}$ & $x^{2}=0.214$ & $p=0.686$ \\
\hline Education (years) & $10.45(4.86)$ & $11.02(3.24)$ & $\mathrm{t}=-0.62$ & $p=0.515$ \\
\hline MMSE (0-30) & $26.90(1.51)$ & $27.46(1.50)$ & $\mathrm{t}=-1.30$ & $p=0.199$ \\
\hline Hoehn and Yahr scale & $2.25(0.63)$ & - & - & - \\
(I/II/III) & & & & \\
\hline Motor UPDRS & $17.05(3.15)$ & - & - & - \\
\hline Disease duration (years) & $5.75(3.52)$ & - & & \\
\hline BDI (0-21) & $5.80(2.87)$ & $5.40(3.71)$ & $\mathrm{t}=0.406$ & $p=0.686$ \\
\hline EHI (1-10) & $9.55(0.76)$ & $9.87(0.73)$ & $\mathrm{t}=-0.822$ & $p=0.415$ \\
\hline & & & & \\
\hline
\end{tabular}

Note: Mean (standard deviations) are given. $\mathrm{N}=$ total number of participants, $\mathrm{M}=$ male, $\mathrm{F}=$ female, MMSE = Mini Mental state Examination, UPDRS = Unified Parkinson's Disease Rating Scale, BDI = Beck Depression Inventory, EHS = Edinburg Handedness Inventory. *Difference is significant at the $p<0.05$ level. 
Table 2 Summary of emotion induction stimulus material

\begin{tabular}{ll}
\hline Emotion & Content (source) \\
\hline Happiness & Funny animals, funny kids, (You Tube, Facebook, and others), \\
Sadness & Disasters, Funeral, Accidents, a dying hospitalized women (IAPS \& IADS) \\
Fear & Murder, Snakes, tornado (IAPS \& IADS) \\
& Child Abuse, Acid attack, Man attacking a women etc., (You Tube, \\
Anger & Facebook, and others ) \\
Surprise & Different wonder activities by human, (You Tube, Facebook, and others) \\
Disgust & Injured and decomposed human body, a burn victim, dirty toilets, bloody \\
& injuries etc., (IAPS \& IADS) \\
\hline
\end{tabular}

Table 3 Mean subjective ratings of emotional stimuli by PD patients and healthy controls.

\begin{tabular}{lllll}
\hline \multirow{2}{*}{ Emotion } & PD & \multicolumn{3}{l}{ HC } \\
\cline { 2 - 5 } & Mean & SD & Mean & SD \\
\hline Happiness & 4.25 & 1.12 & 4.37 & 0.96 \\
Sadness & 3.55 & 1.28 & 3.77 & 0.68 \\
Fear & 3.45 & 0.94 & 3.57 & 1.07 \\
Anger & 3.30 & 1.17 & 3.63 & 0.96 \\
Surprise & 3.85 & 0.75 & 3.90 & 1.12 \\
Disgust & 2.95 & 1.10 & 3.07 & 1.23 \\
\hline
\end{tabular}

Note. Ratings: $1=$ very low, $2=$ low, $3=$ medium, $4=$ high, and $5=$ very high 


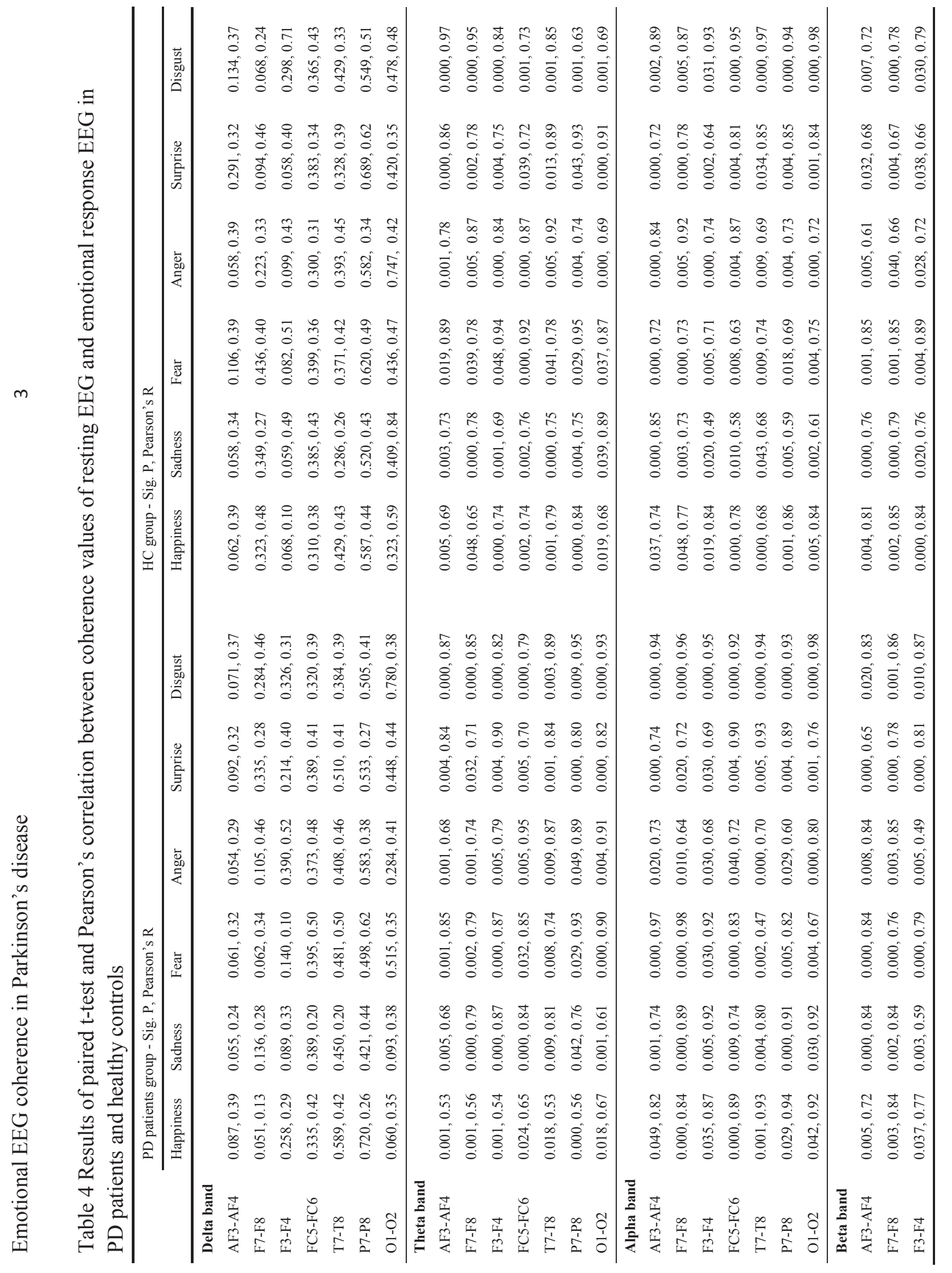




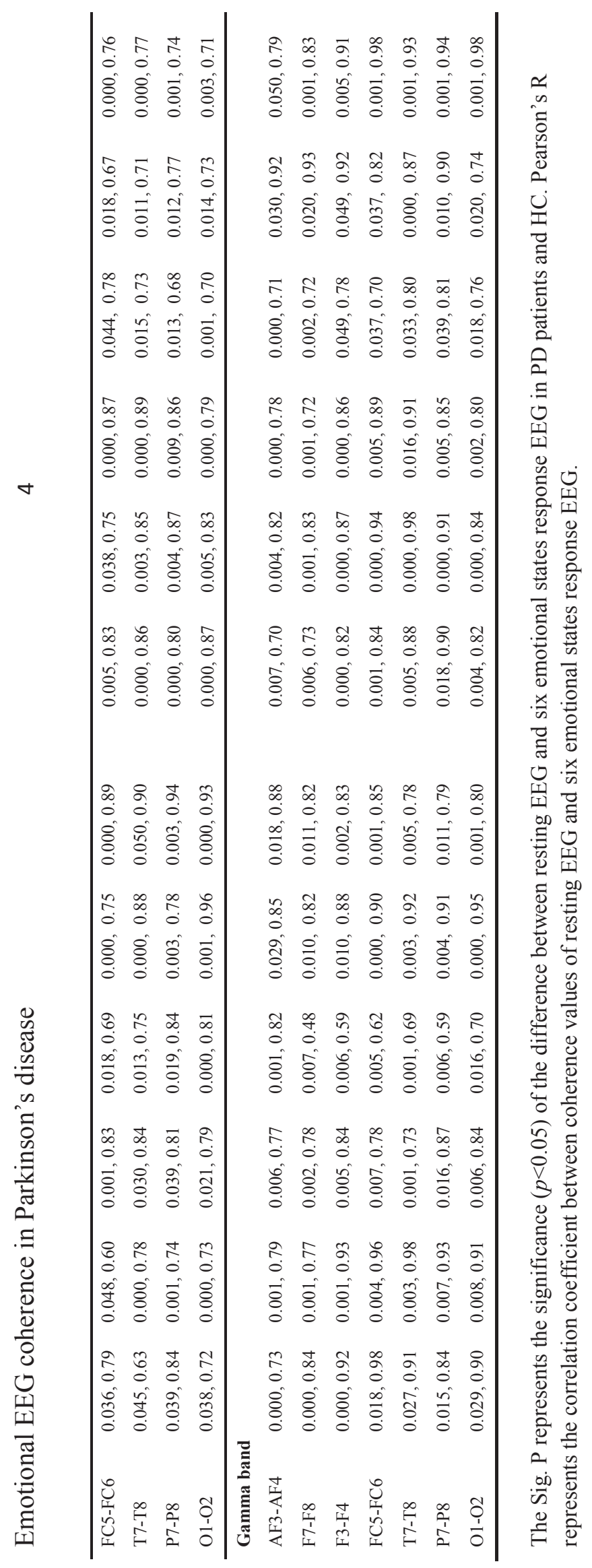




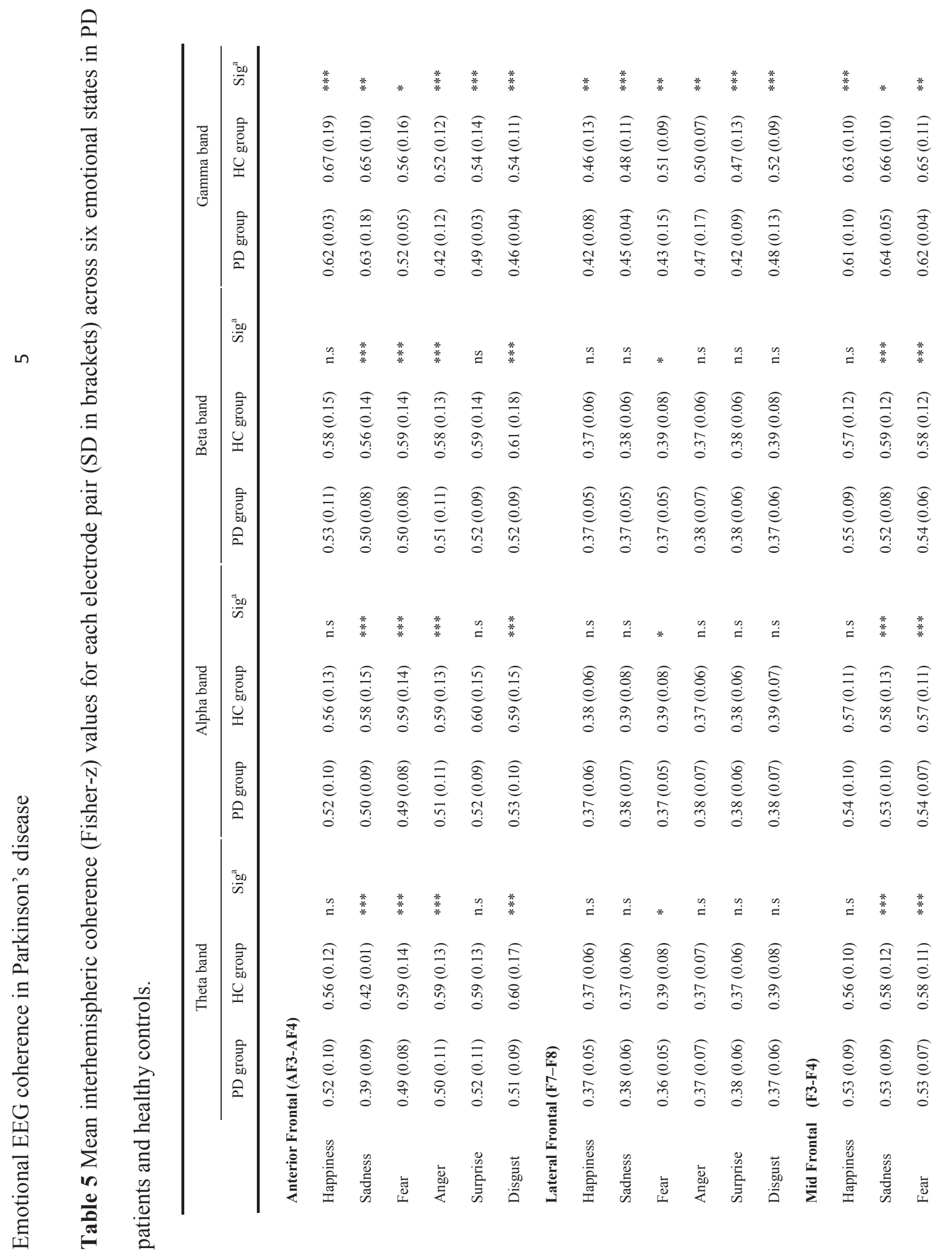




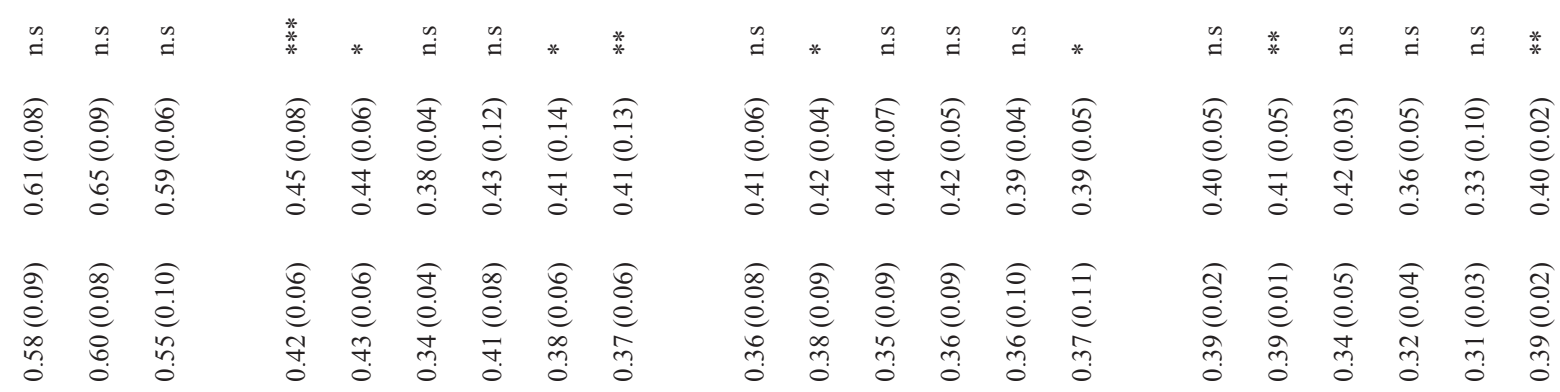



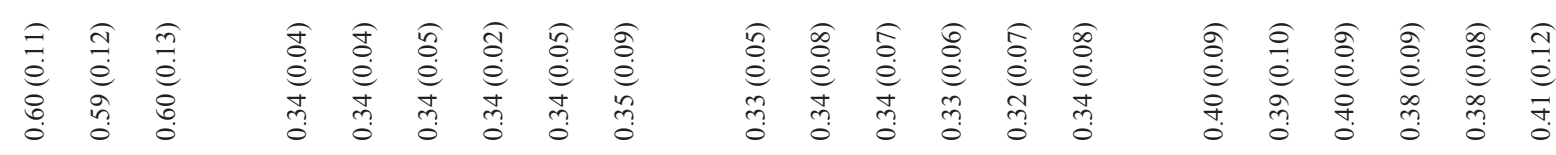

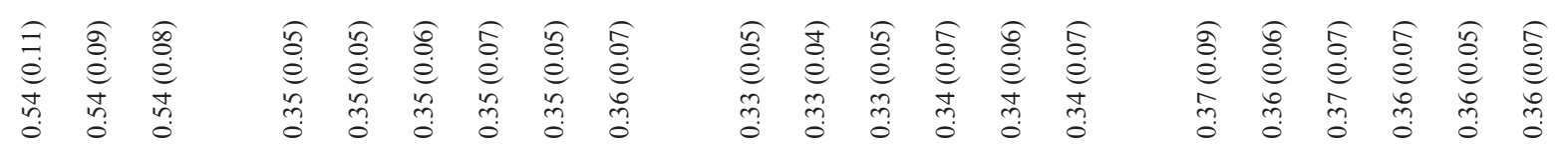

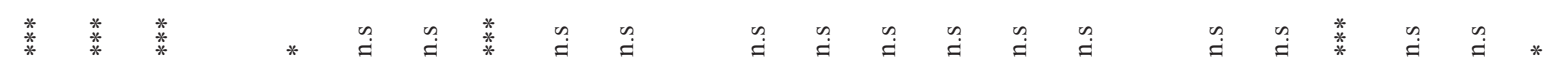

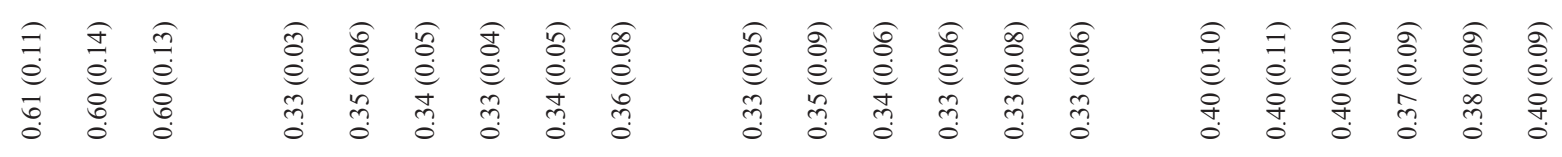

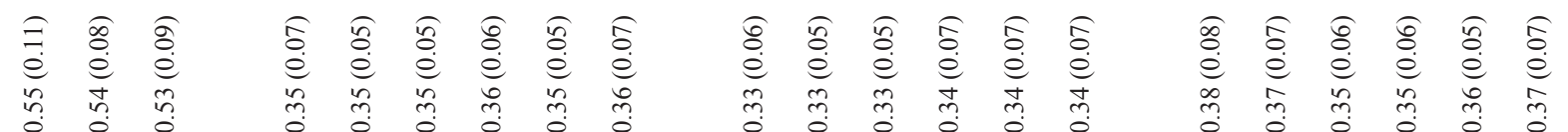



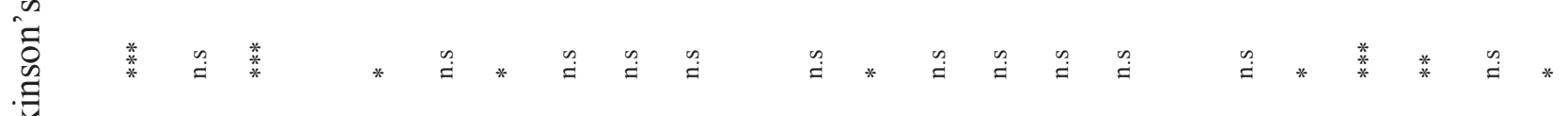

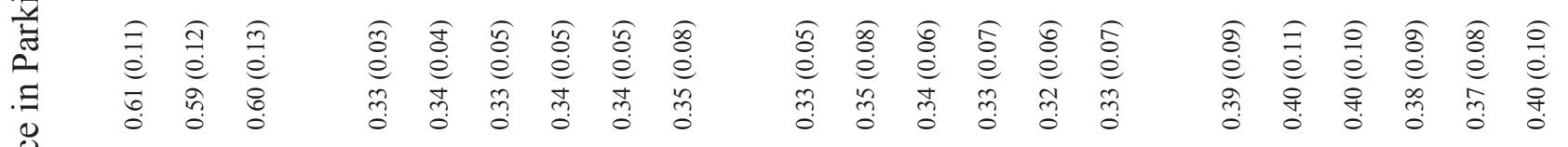

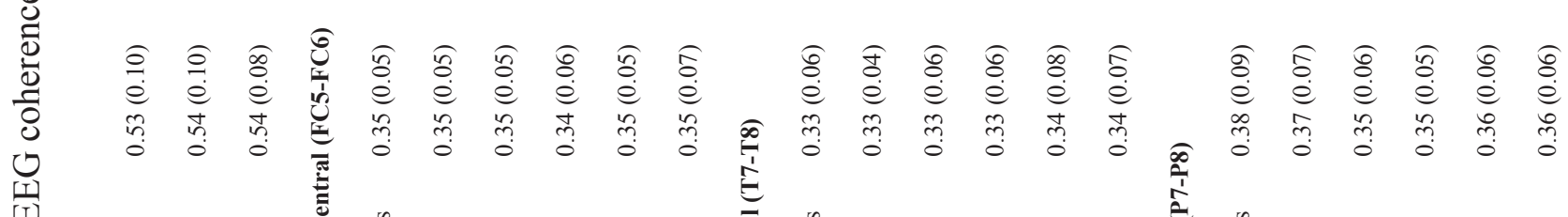

至

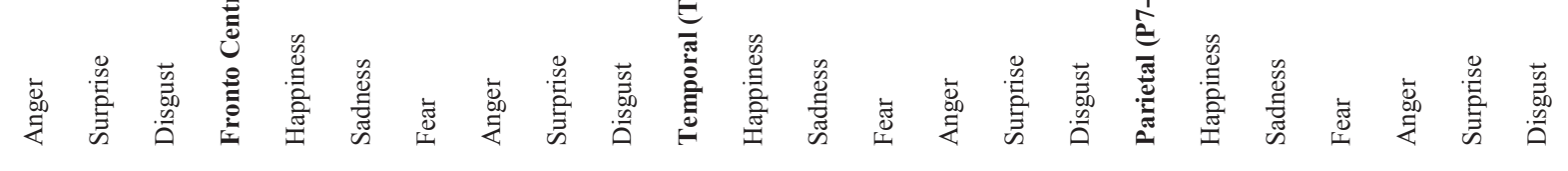




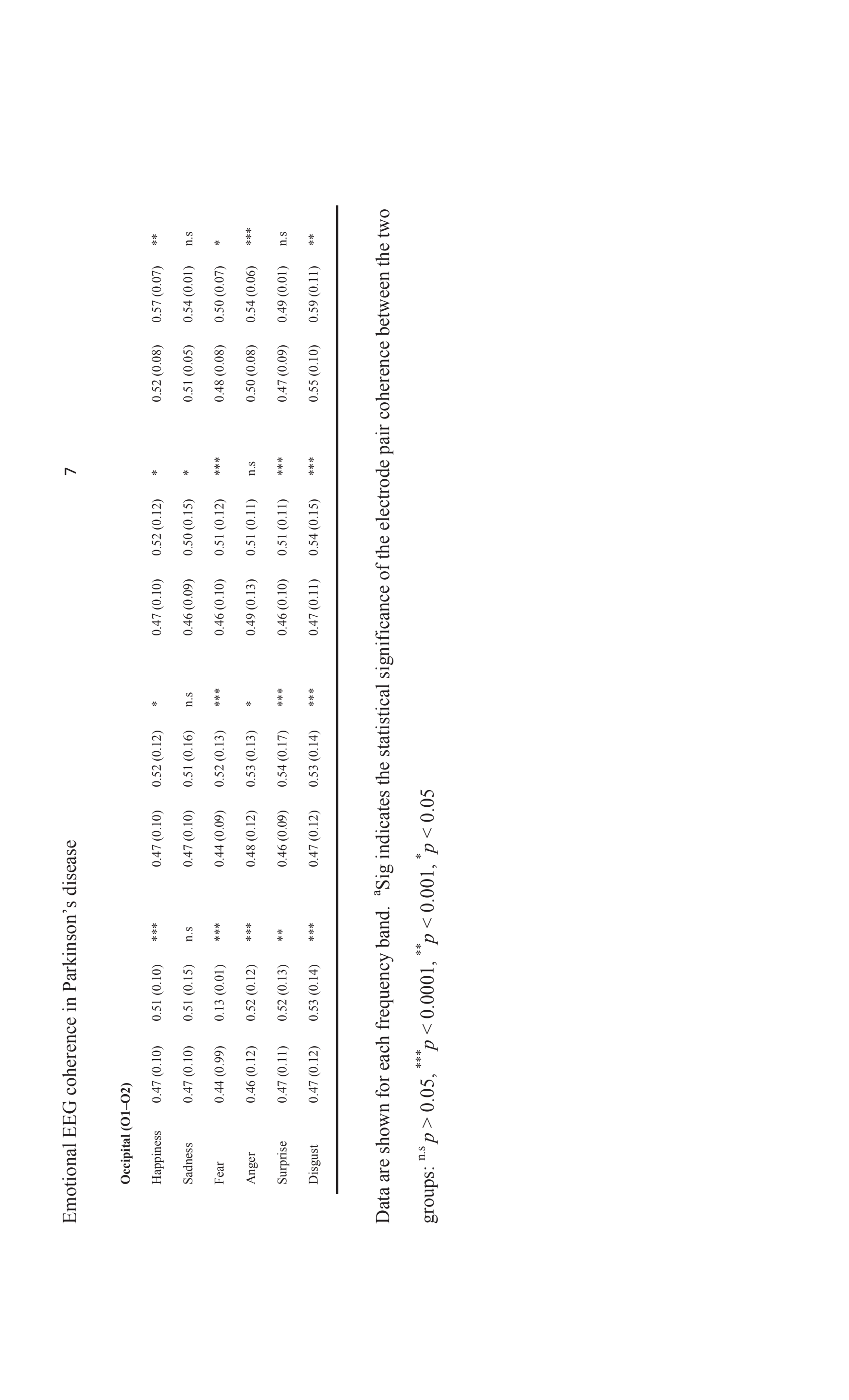

